\title{
Dynamic Fiber-Optic Shape Sensing Using Fiber Segment Interferometry
}

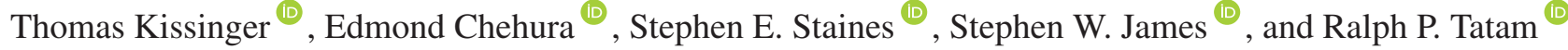

\begin{abstract}
Dynamic fiber-optic shape sensing, often also referred to as curvature or bend sensing, is demonstrated using fiber segment interferometry, where chains of fiber segments, separated by broadband Bragg grating reflectors, are interrogated using rangeresolved interferometry. In this paper, the theory of interferometric curvature sensing using fiber segments is developed in detail, including techniques to infer lateral displacements from the measured differential strain data and methods for directional calibration of the sensor. A proof-of-concept experiment is performed, where four fiber strings, each containing four fiber segments of gauge length $20 \mathrm{~cm}$ each, are attached to the opposing sides of a flexible support structure and the resulting differential strain measurements are used to determine the lateral displacements of a $0.8 \mathrm{~m}$ cantilever test object in two dimensions. Dynamic tip displacement measurements at $40 \mathrm{~nm} \cdot \mathrm{Hz}^{-0.5}$ noise levels over a $21 \mathrm{kHz}$ bandwidth demonstrate the suitability of this approach for highly sensitive and cost-effective fiber-optic lateral displacement or vibration measurements.
\end{abstract}

Index Terms-Condition monitoring, optical fiber sensors, optical interferometry, shape measurement, vibrometers.

\section{INTRODUCTION}

$\mathbf{F}$ IBER-OPTIC shape sensing (FOSS) [1]-[20], allows the curvature, and therefore the shape, of the structure to which a fiber or fiber bundle is attached, to be followed through space and permits the determination of lateral displacements from the curvature measurements. Direct FOSS techniques are based on evaluating the effects due to differential strain as a result of curvature within a single fiber, within a fiber bundle or within the cores of a multicore fiber. In addition to direct FOSS techniques, there are also indirect fiber-optic methods that may be used to deduce the shape of a structure using a number of strain measurements at strategic locations, processed with the help of a detailed mechanical model of the structure using strain-deformation conversion methods [10]. A key advantage of direct FOSS compared to indirect methods is that the measurand, curvature-induced differential strain, is determined directly within the sensor fiber or fiber bundle. Therefore this

Manuscript received July 21, 2017; revised August 24, 2017; accepted August 28, 2017. Date of publication September 11, 2017; date of current version February 24, 2018. This work was supported by the Engineering and Physical Sciences Research Council, U.K., under Grants EP/NO02520/1 and EP/M020401/1. (Corresponding author: Thomas Kissinger.)

The authors are with the Engineering Photonics, Cranfield University, Cranfield MK43 0AL, U.K. (e-mail: t.kissinger@cranfield.ac.uk; e.chehura@ cranfield.ac.uk; s.staines@cranfield.ac.uk; s.w.james@cranfield.ac.uk; r.p. tatam@cranfield.ac.uk).

Color versions of one or more of the figures in this paper are available online at http://ieeexplore.ieee.org.

Digital Object Identifier 10.1109/JLT.2017.2750759 only requires the sensor to follow the shape of the object under test, removing the need for fully established strain transfer between the sensor and the test object, significantly simplifying the sensor attachment, especially for embedded applications. Also, compared to indirect FOSS, temperature sensitivity is greatly reduced in direct FOSS due to the differential nature of the measurement. If the sensor fibers in direct FOSS can be assumed to be at equal temperature or even thermally coupled, such as in multi-core fibers, any temperature-induced drift in the measurement will cancel out during the differential strain calculation.

Prior direct FOSS approaches within single-core single-mode fibers have used long-period gratings [1], [2], [7] or other short-gauge length in-fiber structures, such as in-fiber MachZehnder interferometers [12], [15]. However, in general these approaches, while often achieving extremely high curvature sensitivities, have been primarily designed to be singular curvature sensors. Conversely, distributed FOSS techniques, based on Rayleigh [9] and recently Brillouin scattering [19] within multi-core fibers, are inherently multi-point techniques that can be deployed over long lengths (hundreds of meters), but generally require complex optical setups and achieve only moderate curvature sensitivities. Fiber Bragg gratings [21] (FBGs) are a mature technology that has good multiplexing capabilities. The use of arrays of FBGs co-located at a number of positions along an arrangement of fibers or in the cores of multicore fibers allows FOSS measurements [5], [6] to be conducted. The interrogation of FBGs can be performed using wavelength-division multiplexing (WDM), allowing high-speed measurements $(>\mathrm{kHz})$ of typically several tens of FBGs [22]. Furthermore, much larger numbers of FBG sensors can be interrogated using optical frequency domain reflectometers [23] (OFDRs). While OFDR using FBGs has been used for FOSS [9], [18], it typically achieves lower speeds and has higher uncertainties [24] than WDM approaches. FBG-based FOSS approaches are showing promise in structural health monitoring applications, in areas such as aircraft wings [18], wind turbine blades [13] or within geotechnical structures [8], and in medical applications, such as control of surgical instruments [16], [17]. In this paper the use of this FOSS to measure structural vibrations is presented, therefore further potential applications of FOSS could also be envisaged in this area. This can complement existing laser Doppler vibrometry techniques [25] by removing the need for side access to a structure-under-test and allowing the direction of vibrations to be determined, permitting fiber-optic vibration measurements of internal parts situated within complex machinery. 
Interferometric long-gauge length approaches [3], [4], [11] have also been investigated for FOSS, promising fast and sensitive measurements. However, practical applications of interferometric FOSS appear to have suffered from difficulties in multiplexing sensors and so far interferometric FOSS has only been demonstrated for singular fiber sections. The approach demonstrated in this paper employs fiber segment interferometry (FSI) [26], allowing the spatial resolution of interferometric curvature measurements for the first time. FSI is an emerging technique allowing many interferometric sensor segments to be multiplexed using range-resolved interferometry (RRI) [27], where RRI is designed to exploit the potential that robust, costeffective and highly coherent laser diodes originating in the telecoms industry offer to sensing applications. FSI achieves typical dynamic strain resolutions well below $1 \mathrm{n} \epsilon \cdot \mathrm{Hz}^{-0.5}$ [26], levels that are significantly below commonly used non-interferometric FBG interrogation approaches [22]. In general, interferometric measurements, without further refinements, such as multiwavelength techniques [28], allow only measurements relative to an unknown starting position, however, for the dynamic applications that are targeted in this paper, this is of little consequence. In general, in FOSS, curvature sensitivity scales proportionally with the lateral fiber core distance. Therefore a fiber arrangement mounted onto a flexible support structure that can follow the shape of the object under test, with lateral fiber core spacing of typically several millimeters, offers two orders of magnitude increased curvature sensitivity compared to implementations using multicore fibers, which have typical lateral core spacing of $\approx 50 \mu \mathrm{m}$, in addition to simplified sensor construction. Therefore, the use of a fiber arrangement on a flexible support structure is the approach taken in this work in order to demonstrate the key concepts of direct FOSS using FSI. Future demonstrations using multicore fiber can easily be envisioned and are under investigation.

In this paper, expanding work first reported at the 25th Optical Fiber Sensors Conference, OFS-25 [20], we present a detailed theory of segmented interferometric shape sensing, including methods for sensor calibration, and techniques that allow the inference of lateral displacements from the original differential strain measurements. An experimental proof-of-concept experiment using a cantilever test object is presented, demonstrating the potential of the approach for two-dimensional lateral displacement and vibration measurements.

\section{THEORY}

\section{A. Interferometric Curvature Sensing}

The theory of interferometric curvature sensing is detailed here because it differs from the theory of FBG-based curvature sensing [6], [9], [14]. Interferometric curvature sensing integrates the differential strain over extended path sections, which, as will be shown below, leads to a measurement of the tangent angle difference between the start and end of the sensing section. As illustrated in Fig. 1(a), for a two-dimensional section of constant curvature, defined by curvature radius $R$ or curvature $\kappa=R^{-1}$, it can be seen that for two fiber cores of fixed lateral separation $\delta$, bent along a section of an arc with angle $\psi$ and path

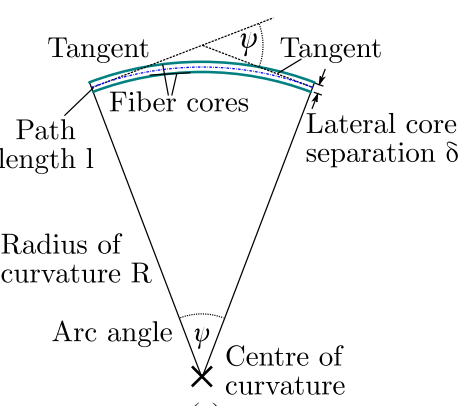

(a)

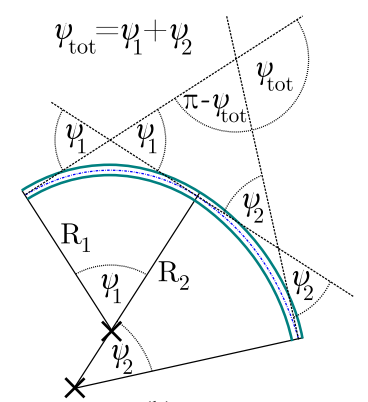

(b)
Fig. 1. An illustration of the geometry for a section of constant curvature (adapted from Blanchard et al. [3]) is shown in (a), while (b) illustrates the integration of differential strain and the resulting addition of the measured angles over two sections with differing curvature.

length $l=\psi \cdot R$, the geometrical path difference between the two fiber cores is given by $\psi \cdot \delta$. Here, the arc angle, $\psi$, is also equal to the angle between the tangents at the start and the end of the curved section [3]. The differential strain $\epsilon_{\text {diff }}$ between the two fiber cores over path length $l$ is then given by:

$$
\epsilon_{\mathrm{diff}}=\frac{\psi \cdot \delta}{l}
$$

Equation (1) also shows that, in general, the curvature sensitivity in FOSS is directly proportional to the lateral core displacement.

In FSI, similar to all interferometric long-gauge length fiber strain sensors [29], the strain sensitivity of a fiber segment, i.e., phase change measured in rad per $\mu \epsilon$, is well-known and is a function of the material properties of the fiber [30]. Therefore, using (1), the resulting arc angle $\psi$ can be expressed as a function of the measured differential phase signal $\Delta \phi$ :

$$
\psi=\frac{\Delta \phi \cdot \lambda_{0}}{\delta \cdot 4 \pi n_{\mathrm{g}} k}
$$

Here, $\lambda_{0}$ is the free-space wavelength of the laser light, $n_{\mathrm{g}}$ is the fiber core group index and $k$ is the fiber strain sensitivity factor. It is important to note that the measurement of the arc angle difference $\psi$ in (2) is independent of the sensor gauge length $l$. Using $l=\psi \cdot R=\psi \cdot \kappa^{-1}$, the curvature sensitivity $\Delta \phi \cdot \kappa^{-1}$ is then given by:

$$
\frac{\Delta \phi}{\kappa}=\frac{\delta \cdot l \cdot 4 \pi n_{\mathrm{g}} k}{\lambda_{0}}
$$

Furthermore, is was first observed by Blanchard et al. [3] that for more complex two-dimensional paths, an interferometric curvature sensor will integrate all angular changes, with the measured result still being equal to the difference in the angle between the start and the end tangents of the path. This is illustrated in Fig. 1(b), which shows the concatenation of two sections with different curvature radii $R_{1}$ and $R_{2}$ and arc angles $\psi_{1}$ and $\psi_{2}$. It is illustrated how the angle $\psi_{\text {tot }}$ between the start and end tangent of the complete path is the sum of $\psi_{1}$ and $\psi_{2}$. This holds for both negative and positive curvatures, i.e., negative and positive arc angles, and also for straight sections, where the arc angle is zero. This geometrical argument can be extended to any complex two-dimensional path that is continuously differentiable, i.e., where a tangent can be defined 
anywhere, and which can thus be described by the concatenation of curvature sections with curvature radii $R_{i}$ and arc angles $\psi_{i}$ of index $i$. Therefore, an interferometric measurement of the differential strain of two fiber cores held at constant lateral separation $\delta$ over such a complex path will be proportional to the sum of the arc angles $\sum \psi_{i}$ multiplied by $\delta$. In effect, an interferometric measurement will therefore be a direct measurement of the difference in the angle between the start and end tangents of the path. Additionally, if the interferometric measurement is range-resolved, such as in FSI, the tangential angles, i.e., the local arc angles, along the fiber path are effectively sampled after each sensor section.

The above analysis is initially valid only for curvature measurements in a two-dimensional plane. To extend this to three dimensional paths, it has to be assumed that no twist or torsion is introduced axially along the fiber core arrangement, which ensures that the two radial curvature components are independent of each other and that a change in curvature in one direction does not introduce differential strain in the other. We therefore limit our analysis to the three dimensional case without twist, as the generalized three-dimensional description incorporating twist requires further mathematical analysis that is beyond the scope of this paper.

\section{B. Lateral Displacement Determination}

In order to obtain the desired lateral displacement information along the fiber path, the measured arc angles, also referred to as slope angle in a mechanical engineering context, need to be integrated along the sensor path. As discussed in the last paragraph an FSI curvature measurement effectively results in the sampling of the local slope angle along the fiber path. In order to obtain the lateral displacements, an assumption has to be made on the slope angle function between the sampling points to allow integration. Without prior knowledge of the structure, a linear interpolation of the slope angles between the sampling points is the simplest solution, while more complex approaches, such as cubic spline interpolation, are also possible. If there is prior knowledge of the expected slope angle function, for example from a mechanical model of the structure under test, then this model can also be used to fit the measured slope angle data, which would improve the accuracy of the displacement determination if the assumption on which the mechanical model is based are correct. In this paper, we will compare linear and cubic spline interpolation approaches with the model-fit approach for an intermediately-loaded cantilever. Here, for a cantilever of length $l$ with a load at position $x=a$ along the cantilever position coordinate $x$, the slope angle function $\psi(x)$ is given by [31]:

$$
\psi(x)=\left\{\begin{array}{cc}
\frac{P}{2 E I} x(2 a-x) & 0 \leq x \leq a \\
\frac{P}{2 E I} a^{2} & a \leq x \leq l
\end{array}\right.
$$

In (4), $P$ is the load, while $E$ is the Young's modulus and $I$ is the moment of inertia of the beam. Note that for displacement calculations using (4), the factor $\frac{P}{2 E I}$ does not need to be known a priori but can be fitted to the measured slope angle data if the position $a$ of the load is known.
Furthermore, it is interesting to discuss the challenge of shape sensing for large structures. For the mechanical model of the simple but representative case of an end-loaded cantilever of length $l$, it can be shown [3], [31] that the slope angle $\psi$ between start and end of the cantilever as a function of the cantilever tip displacement $d$ is given by:

$$
\psi=\frac{3 d}{l}
$$

Inserting (5) into (1) results in:

$$
\epsilon_{\mathrm{diff}}=\frac{3 d \cdot \delta}{2 l^{2}}
$$

From this example it can be seen that the differential strain resulting for a given cantilever tip displacement $d$ is proportional to $l^{-2}$, i.e., the tip displacement sensitivity reduces quadratically with increasing cantilever length. This illustrates that in fiberoptic shape sensing for large structures achieving high strain sensitivity of the interrogation system is of paramount importance.

\section{SETUP}

\section{A. Optical Setup and Signal Processing}

The optical setup used in this work is illustrated in Fig. 2(a), employing the fully-enclosed interrogation unit, also pictured in Fig. 2(e). The optical setup of the interrogation unit consists of an InGaAs photo detector, a fiber-optic circulator and an Eblana EP1550-0-NLW-B06-400FM laser diode with $420 \mathrm{kHz}$ line width, emitting at a center wavelength of $\lambda_{0}=1548.1 \mathrm{~nm}$. To allow RRI signal processing [27], the laser is injection current modulated with a sinusoidal waveform that results in a peak-to-peak wavelength modulation amplitude of $\approx 0.22 \mathrm{~nm}$ as measured using an optical spectrum analyzer.

The light leaving the interrogation unit is then split, using a $4 \times 1$ coupler, into four fiber strings A, B, C and D. In each fiber string five low-reflectivity, broadband FBGs are inscribed resulting in fiber segments of $20 \mathrm{~cm}$ length, with FBG reflectors labeled A1...D5, located at positions shown in Fig. 2(a). The details of the FBG inscription process is detailed in the next section. In each fiber string, the desired interference signals occur between the fiber tip Fresnel reflection and the five FBG reflectors and any interference signals between individual FBG reflectors are undesired. Each fiber string has a unique distance between fiber tip and the set of FBG reflectors, incrementing per fiber string by the value of $\approx 4 \mathrm{~cm}$.This assigns unique ranges to all the desired FBG signals, for example $4 \mathrm{~cm}$, $24 \mathrm{~cm}, 44 \mathrm{~cm}$, etc. for the reflectors of string $A$ and $16 \mathrm{~cm}$, $36 \mathrm{~cm}, 56 \mathrm{~cm}$, etc. for the reflectors of string D. This allows all fiber strings to be interrogated simultaneously using a single interrogation unit with a single laser and photo detector, where $\Delta r \approx 4 \mathrm{~cm}$ is then the effective range separation that needs to be resolved by the RRI signal processing to recover signals from all four fiber strings present. Furthermore, undesired interference signals occurring between the individual FBG reflectors themselves will fall into ranges of $20 \mathrm{~cm}, 40 \mathrm{~cm}$, etc. and therefore do not intrude into any of the desired signal ranges. Additionally, in order to prevent undesired interference signals across 
(a) Optical Setup

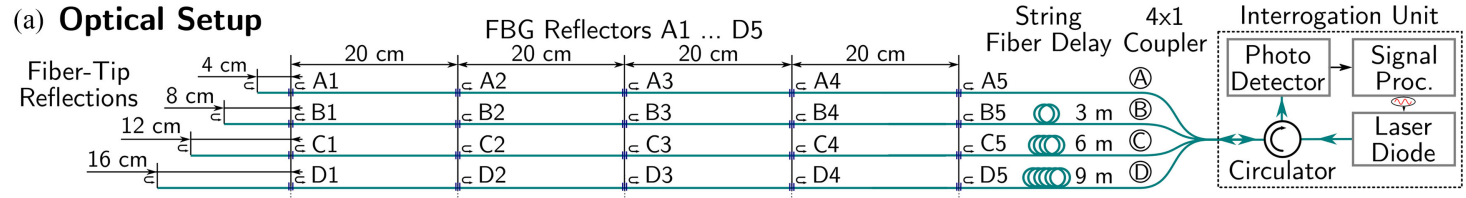

(b) Rod Side View

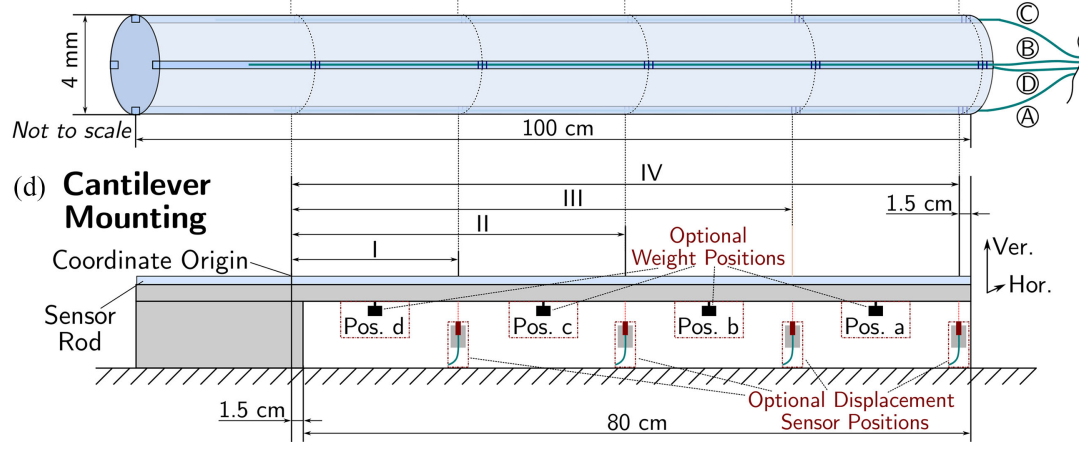

(c) Rod Cross Section

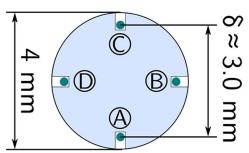

(e) Interrogation Unit

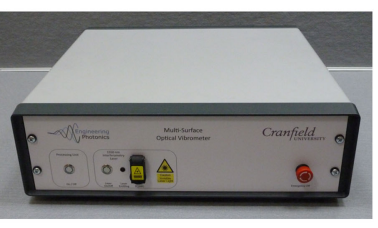

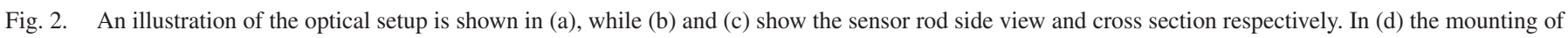
the sensor rod onto the cantilever test object is drawn, while (e) shows a picture of the full-enclosed interrogation unit used.

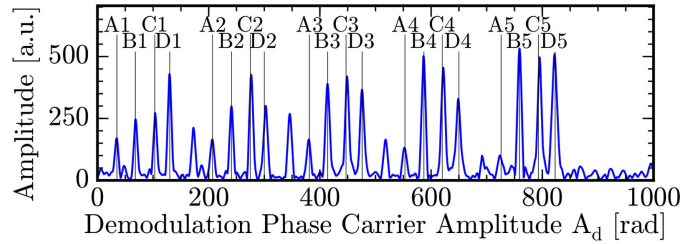

Fig. 3. Plot of the signal amplitude as a function of the demodulation phase carrier amplitude $A_{\mathrm{d}}$, with the peaks corresponding to the desired signals from reflectors A1 to D5 labeled.

fiber strings, a unique fiber delay line per string at $3 \mathrm{~m}$ intervals has been inserted after the coupler, moving cross-string interference signals well out-of-range. Fig. 3 shows the evaluated return signal, calculated over a single modulation period, as a function of the demodulation phase carrier amplitude $A_{\mathrm{d}}$ [27], which is approximately proportional to the optical path difference, i.e., range, of the interference signals present. The values of $A_{\mathrm{d}}$ where the evaluation for each peak takes place are labeled in Fig. 3 with vertical lines and with the reflector labels corresponding to the labels in Fig. 2(a). Fig. 3 clearly demonstrates how in FSI signal peaks at desired ranges can be separated from undesired sources at other ranges. In this setup, for the effective range separation $\Delta r \approx 4 \mathrm{~cm}$, the peak-to-peak sinusoidal wavelength modulation amplitude of $0.22 \mathrm{~nm}$ leads to $A_{\mathrm{d}}$ values incremented by $\approx 33 \mathrm{rad}$, as evident in Fig. 3 . This is almost a factor of three more than the value of $A_{\text {min }}$ required according to the spatial resolution calculation [27] for a specified baseband suppression value of $50 \mathrm{~dB}$ at $\sigma=0.05$, where $\sigma$ is the Gaussian window width parameter used in the RRI signal processing. This leaves some leeway to improve the spatial resolution of this shape sensing approach for future, optimized implementations. Additionally, spatial resolution could also be improved by increasing the sinusoidal wavelength modulation amplitude of the laser.

The signal processing is implemented on an Altera Cyclone-IV field programmable gate array (FPGA), which controls and synchronizes both the laser modulation and the demodulation of the photo detector signals. Furthermore, in the fully-enclosed interrogation unit pictured in Fig. 2(e), a regular $\mathrm{PC}$ is integrated in the unit and used to capture the data processed by the FPGA and controls the measurement via a PCI express bus implementation using the Riffa framework [32]. In contrast to previous RRI implementations [26], [27] a lowercost Thorlabs ITC102 integrated laser driver module is used to sinusoidally modulate the laser injection current at a modulation frequency of $49 \mathrm{kHz}$. This laser driver exhibits higher inherent drift of the modulation signal phase than drivers used in previous implementations, therefore, in order to simplify the calibration of the signal processing time delay [27], only a single window per modulation and not the dual-window function previously described is used, which relaxes the signal processing time delay setting accuracy, but halves the signal-to-noise ratio of the detection. Also, due to the nonlinearity of the laser modulation process, higher order nonlinear corrections of the modulation waveform of $5.7 \%$ for the first harmonic and $0.7 \%$ for the second harmonic, as determined by visual inspection of the phase carrier amplitude versus signal processing delay maps, were applied to the demodulation carriers in addition to the correction of parasitic intensity modulation [27].

\section{B. FBG Inscription and Sensor Rod Fabrication}

In FSI, broadband reflectors are required that return signals over all conceivable temperature and strain conditions. The reflectors can be very weak, with reflectivities down to parts-permillion (ppm) possible [26]. However, in the setup of Fig. 2(a), the $1 \times 4$ coupler, which is passed twice, effectively introduces an optical power loss of a factor of 16 compared to FSI in a single fiber [26], requiring the use of correspondingly stronger gratings. In this work, broadband FBGs are inscribed in photosensitive Fibercore PS1250/1500 fiber using a frequency-quadrupled $\mathrm{Nd}$-YAG laser operating at $266 \mathrm{~nm}$ according to a method previously described by Wang et al [33]. Here, a slit of $0.2 \mathrm{~mm}$ width 


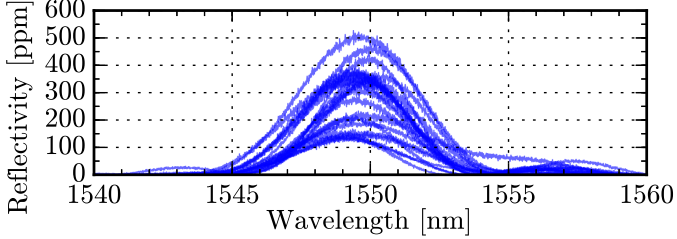

Fig. 4. Overlaid plot of the reflection spectra all 20 FBGs used.

placed between the phase mask and the fiber is used to limit the length of the FBG and thus increase the reflection bandwidth. An overlay plot of the reflection spectra of all 20 FBGs used, as measured by a Luna OBR 4400 reflectometer, which uses an OFDR approach [23] to obtain multiple FBG spectra at different ranges, is shown in Fig. 4. Here it can be seen that the produced gratings have reflectivities of typically $130 \mathrm{ppm}$ to $500 \mathrm{ppm}$ and bandwidths of approximately 4 to $5 \mathrm{~nm}$ full-width half maximum.

Fig. 2(b) shows how the fiber reflectors are co-located on the four opposing sides of the PTFE sensor rod of $4 \mathrm{~mm}$ outer diameter. Channels were cut manually in the rod to securely locate the fiber on the rod, with the rod cross section illustrated in Fig. 2(c). The channels are approximately $0.6 \mathrm{~mm}$ deep, resulting in a nominal lateral fiber core spacing $\delta$ of $\approx 3.0 \mathrm{~mm}$. This spacing results in a slope angle sensitivity of $\Delta \phi \cdot \psi^{-1}=487$ rad of measured differential phase change per degree of applied slope angle change, according to (2). This calculation uses the approximate values of $n_{\mathrm{g}}=1.45$ and $k=0.79$ [34] for the type of photosensitive fiber used in this work. As the fabrication of the channels was a manual process, control of the channel depth was limited, which results in some uncertainty for the value of $\delta$ and thus for the sensitivity $\Delta \phi \cdot \psi^{-1}$, which could be as high as $10 \%$. However, in future implementations we anticipate that by using a custom extruded PTFE profile instead of the manual fabrication process of the sensor rod, these uncertainties will become negligible.

The fibers are attached to the rod by filling the channels with a cyanoacrylate adhesive after inserting the fibers. The measured differential strain signals are then obtained by subtraction of the signals from the interferometers formed in strings $\mathrm{A}$ and $\mathrm{C}$ for the vertical direction and from those in strings $\mathrm{B}$ and $\mathrm{D}$ for the horizontal direction. Throughout this paper, we will refer to sensor Section I to IV, all originating at the cantilever mount, as shown in Fig. 2(d), instead of referring to measurements from individual fiber segments, as this simplifies the mathematical treatment by relating all measurements to the common, stationary origin. The measurements for sensor Sections I to IV then sample the cantilever slope angle along the cantilever, which can be easily obtained by subtracting the signals from the corresponding end reflectors from the reflector signals A1 to D1 at the cantilever origin. As can be seen in Fig. 2(d), the sensor rod is mounted on a cantilever test object, a $15 \times 15 \mathrm{~mm}$ square bar of 316 stainless steel. Here, the relevant cantilever length is $80 \mathrm{~cm}$. The last reflectors in each string, A5 to D5, are recessed by $1.5 \mathrm{~cm}$ from the cantilever tip so that the sensor rod can be correctly attached to follow the cantilever shape. As discussed previously, interferometric curvature sensing only requires the

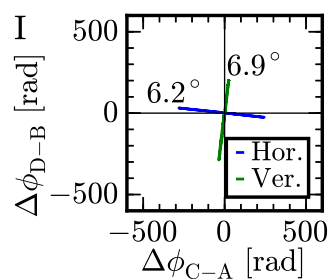

(a)

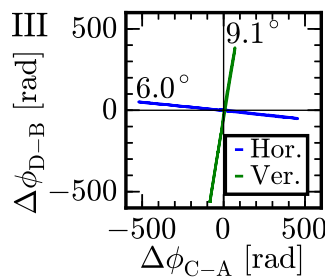

(c)

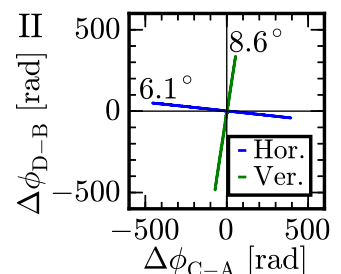

(b)

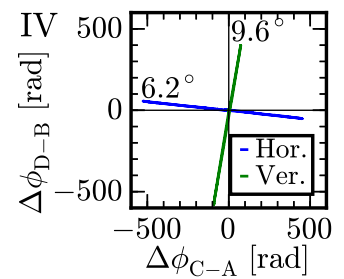

(d)
Fig. 5. The sensor responses to horizontal and vertical movements in the optical table coordinate system for Sections I to IV are shown in plots (a) to (d) respectively. Here the annotated numerical values are the extracted angles between the measurements and the optical table horizontal/vertical axis.

sensor rod to follow the structure under test and does not necessitate fully established strain transfer between the sensor rod and the structure. Therefore, to demonstrate this point in this work, the sensor rod was attached to the cantilever using $3 \mathrm{M}$ 431 aluminum tape only, where full strain transfer cannot be expected.

\section{RESUlTS AND DisCUSSION}

\section{A. Directional Calibration}

In this work, good control of the sensor directional alignment is difficult to achieve while laying down the sensor rod on the test object due to its circular shape, and therefore the orientation of the sensors axes needs to be calibrated. However, we anticipate that in future implementations, for example by using an custom extruded plastic profile incorporating a directional guide into the sensor rod, such as a flat sensor underside, there will be no requirement for a directional calibration.

In order to calibrate the directional response of the sensor, the cantilever is subjected to a purely horizontal and a purely vertical movement in optical table coordinates, by moving the cantilever tip manually along an appropriate guide rail. The results from these measurements are shown in Figs. 5(a) to (d) for the four sensor Sections I to IV, respectively. In all cases, measurements for both horizontal and vertical movements are shown on the same plot. Here, the phase signal due to differential strain in strings $\mathrm{C}$ and $\mathrm{A}, \Delta \phi_{\mathrm{C}-\mathrm{A}}$, which is the nominally vertical axis of the sensor, see Fig. 2(c), is plotted on the x-axis, while the nominally horizontal measurement $\Delta \phi_{\mathrm{D}-\mathrm{B}}$ between strings $\mathrm{D}$ and $B$ is plotted on the $y$-axis. The values of the angle, obtained from a linear regression fit of the measurements, between each nominal sensor axis and the horizontal or vertical axis in optical table coordinates are also annotated in Fig. 5. It can be seen in Fig. 5 that the angular orientation is misaligned with the optical table axes by $\approx 6^{\circ}$ to $10^{\circ}$, where the most likely cause for the 

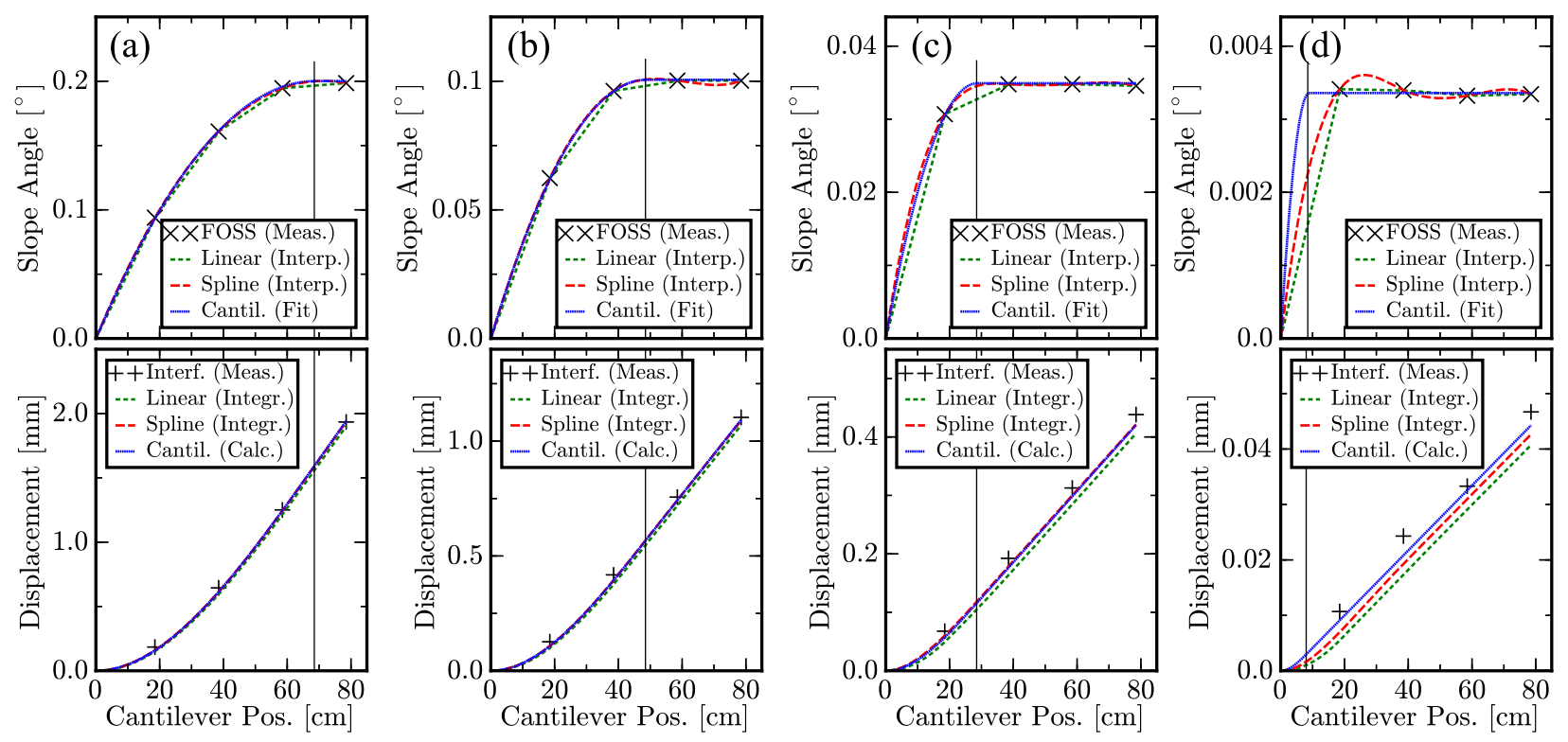

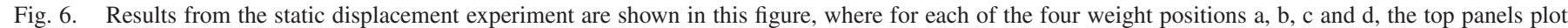

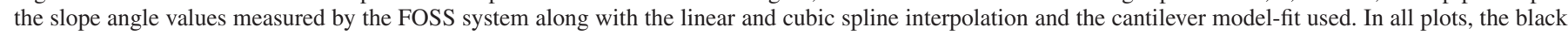

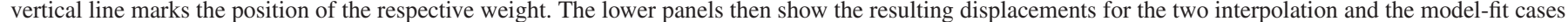
as well as data points from the independent interferometric verification measurement. Also note the change in y-axis scale from (a) to (d).

differences in misalignment is a small amount of twist of the sensor rod. Furthermore the angle between the sensor axes may not be exactly $90^{\circ}$, which may be a result of the manual production process of the sensor rod where it was difficult to maintain the perpendicularity of the channels over the length of the sensor rod. Nevertheless, the linearity of the results in Fig. 5 show the quality of the measurements that can be obtained in principle. In the remainder of the paper, the directional misalignment was corrected by applying a standard Euclidean rotation matrix on the recorded signals $\Delta \phi_{\mathrm{C}-\mathrm{A}}$ and $\Delta \phi_{\mathrm{D}-\mathrm{B}}$, with correction rotation angles of $6.6^{\circ}, 7.4^{\circ}, 7.6^{\circ}$ and $7.9^{\circ}$ for Sections I, II, III and IV, respectively. For each section, these correction angles are simply the average of the corresponding values annotated in Fig. 5. The signals resulting from this rotation are then denoted by $\Delta \phi_{\mathrm{Ver}, \text { Sec }}$ and $\Delta \phi_{\mathrm{Hor}, \text { Sec }}$ for the vertical and horizontal signals in optical table coordinates, where Sec denotes Sections I, II, III and IV.

\section{B. Comparison of Static Displacements with Theory}

As discussed in Section II-B, the interferometric FOSS approach proposed in this paper effectively measures the cantilever slope angles, sampled at the end points of the fiber Sections I, II, III and IV in Fig. 2(d). In order to obtain the desired displacement function of the cantilever, the slope angle function between the sample points needs to be interpolated or fitted to the cantilever model to allow the integration of the slope angle function to yield the displacement function.

In order to verify the accuracy of the FOSS system for the prediction of the desired displacement, we have conducted a series of experiments using a test weight of $1.05 \mathrm{~kg}$ sequentially attached to the cantilever at four positions a, b, c and d, where these positions were determined to be 68.7, 48.7, 28.7 and $8.9 \mathrm{~cm}$, respectively, from the cantilever mount in Fig. 2(d). The predicted displacement measurements from the FOSS system were then verified independently using an external interferometric displacement sensor [27], [35], also based on the RRI technique, which was placed below the cantilever pointing upwards, at positions corresponding to the endpoints of Sections I, II, III and IV, as illustrated in Fig. 2(d).

The crosses in the upper panels in Fig. 6(a) to (d) represent the vertical slope angle measurements using the FOSS system as a function of position along the cantilever for the weight placed at positions a, b, c and d, respectively, averaged over $1 \mathrm{~s}$ and calculated from the rotated differential strain measurements $\Delta \phi_{\text {Ver, I }}$ to $\Delta \phi_{\text {Ver,IV }}$ for Sections I to IV using the nominal slope angle sensitivity value of $487 \frac{\mathrm{rad}}{\mathrm{o}}$ discussed in Section III-B. In all cases in the upper panels in Fig. 6 it can be seen that the slope angle is mostly constant beyond the respective weight position, which is marked by the black vertical lines, as would be expected from (4). Furthermore, the linear and cubic spline interpolation traces between these measurement points are also shown in the upper panels of Fig. 6, along with the visual fit to the cantilever model of (4). Here, the same value of $\frac{P}{2 E I}=0.0074 \mathrm{~m}^{-2}$ could be used to fit the cantilever model to the measured slope angle data points for all four positions of the weight.

The lower panels in Fig. 6 plot the vertical cantilever displacement as a function of position along the cantilever determined from the two interpolation approaches and the cantilever modelfit. Additionally, the crosses in the lower panels in Fig. 6 show the data points for the independent interferometric verification measurement. Note that the y-axis scales in all plots change going from Fig. 6(a) to (d). It can be seen in the lower panel in Fig. 6 that there is excellent agreement between the interferometric verification measurement and the interpolation and model-fit approaches in Fig. 6(a) and that the agreement gets progressively worse going towards Fig. 6(d). In all cases, there 


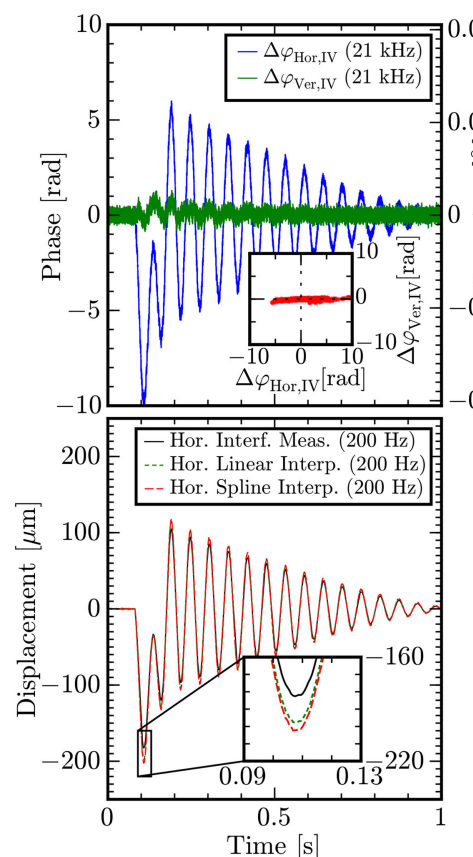

(a)
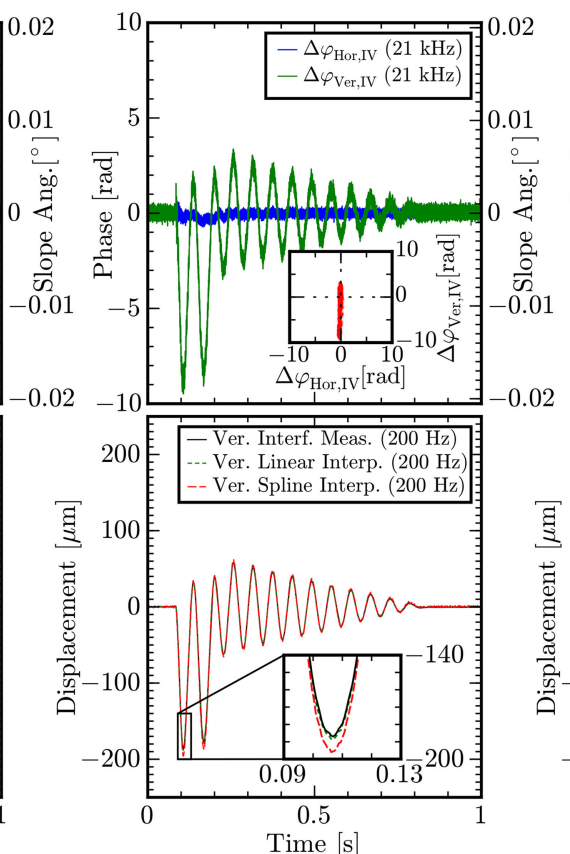

(b)
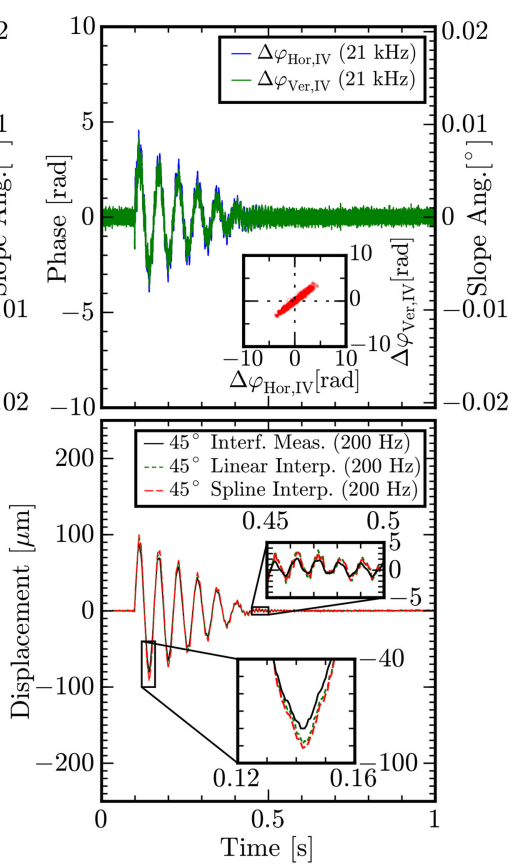

(c)

Fig. 7. Measurement results recorded over $1 \mathrm{~s}$ for a horizontal, vertical and diagonal $\left(\approx 45^{\circ}\right)$ cantilever tip vibration are shown in $(\mathrm{a})$, (b) and (c), respectively. The top panels plot the rotated differential strain phase signals $\Delta \varphi_{\mathrm{H} \text { or, IV }}$ and $\Delta \varphi_{\mathrm{Ver}, \mathrm{IV}}$ for the horizontal and vertical directions, with the corresponding slope angles scale on the secondary y-axis, over the full measurement bandwidth of $21 \mathrm{kHz}$ as a function of time, while the insets plot the two signals against each other. The lower panels then plot the extracted displacement of the cantilever tip for both linear and cubic spline interpolations, now low-pass filtered at a $200 \mathrm{~Hz}$ bandwidth, along with an interferometric verification measurement, with the insets showing zoomed versions of these plots.

is an underestimation of the displacement, which is worst for the linear interpolation, improving for the cubic spine interpolation and the best agreement, as expected, can be achieved for the cantilever model. Looking again at the upper panels in Fig. 6 it can be seen that the expected slope angle function varies more smoothly the further the weight is placed towards the cantilever tip, i.e., more abrupt variation in slope angle occur when the weight is positioned closer to the cantilever origin. This is the reason that the interpolation is getting progressively less able to predict the actual displacements going from Fig. 6(a) to (d). Also, there are higher uncertainties in the cantilever model, such as the influence of the mounting forces, when the weight is placed close to the cantilever origin, explaining the observed discrepancies in Fig. 6(d). Nevertheless, it can be seen in Fig. 6 that, at least for smoothly varying curvature profiles, the FOSS system can be used to measure the cantilever displacement well, even without any knowledge of the structure, as demonstrated by the results for the linear and cubic spline interpolation. In the case of more rapidly varying slope functions, such as Fig. 6(d), it is expected that an increase in the spatial resolution of the FOSS system would improve the displacement measurements.

\section{Vibration Measurements}

To demonstrate the potential of the proposed approach for directional, lateral vibration measurements, a series of experiments was conducted where vibration of the cantilever was manually excited along a guide rail that could be set at an adjustable angle relative to the optical table. Furthermore, as in the previous experiment, an interferometric verification measurement was conducted, with the interferometer pointing in the same direction as the guide rail and using a small reflector attached to the cantilever at the appropriate angle. The results of these measurements for sensor Section IV, corresponding to lateral vibrations near the cantilever tip in Fig. 2(d), are shown in Fig. 7, where Fig. 7(a), (b) and (c) plot results for a horizontal, vertical and diagonal $\left(\approx 45^{\circ}\right)$ excitation, respectively. In Fig. 7 , the top panels of each plot show the signals $\Delta \varphi_{\mathrm{Hor}, \mathrm{IV}}$ and $\Delta \varphi_{\mathrm{Ver}, \mathrm{IV}}$ acquired at the full interferometric measurement bandwidth of $21 \mathrm{kHz}$ over $1 \mathrm{~s}$, where $\Delta \varphi_{\mathrm{Hor}, \mathrm{IV}}$ and $\Delta \varphi_{\mathrm{Ver}, \mathrm{IV}}$ were obtained by rotating the raw differential strain phase signals at an angle of $7.9^{\circ}$ as described in Section IV-A. The secondary y-axis in the upper panels in Fig. 7 also plots the corresponding slope angles, calculated with the previously used sensitivity value of $487 \frac{\mathrm{rad}}{\circ}$. It can be seen in the upper panels in Fig. 7 that for Fig. 7(a) and (b) the vibration occurs mostly in the $\Delta \varphi_{\mathrm{Hor}, \mathrm{IV}}$ and $\Delta \varphi_{\mathrm{Ver}, \mathrm{IV}}$, respectively, while in Fig. 7 (c) both $\Delta \varphi_{\mathrm{Hor}, \mathrm{IV}}$ and $\Delta \varphi_{\mathrm{Ver}, \mathrm{IV}}$ are of approximately equal magnitudes as expected for a diagonal vibration. The insets in the upper panels of Fig. 7 plot $\Delta \varphi_{\text {Ver,IV }}$ directly against $\Delta \varphi_{\mathrm{Hor}, \mathrm{IV}}$, highlighting that the direction of the vibration can be easily identified in this way.

The lower panels in Fig. 7 show the resulting displacements, determined using the linear and cubic spline interpolation, as a function of time along with the external interferometric verification measurement, which measures the displacement at the endpoint of Section IV in Fig. 2(d), with the verification interferometer pointing in the appropriate vibration direction. To show the high quality of the FOSS measurements presented in Fig. 7, the slope angle measurements used for the calculation 
of the interpolated displacement data were low-pass filtered at a bandwidth of $200 \mathrm{~Hz}$. Unlike the static case shown in Fig. 6, the cantilever model of (4) cannot be used for the determination of time-resolved displacements because even the fundamental vibration mode might differ from the static cantilever model and additionally several higher order mode shapes could furthermore be present. Generally, the plots in the lower panels of Fig. 7 show good agreement of the determined displacements from the FOSS system with the interferometric verification measurements. The insets in the lower panels in Fig. 7 zoom in on the regions near the highest vibration amplitudes, showing that the discrepancies are at levels of up to $\approx 10 \%$. The discrepancies are smaller for the vertical vibration in Fig. 7(b) compared to the horizontal vibration shown in Fig. 7(a), where it is thought that the uncertainty in the knowledge of the sensor rod channel depth causes a proportional uncertainty in the slope angle sensitivity $\Delta \phi \cdot \psi^{-1}$, as discussed in Section III-B. Furthermore, there is an additional inset in Fig. 7(c) showing the zoomed plot of a cantilever excitation of a frequency of $\approx 100 \mathrm{~Hz}$ and a peak-to-peak amplitude of $\approx 3 \mu \mathrm{m}$. Here, it can be seen that this signal can be measured by the FOSS system with reasonable agreement, demonstrating the suitability of this approach even for small amplitude, high frequency vibration signals.

The standard deviation of the noise exhibited in the phase signals shown in the top panels of Fig. 7 amounts to $\approx 0.17 \mathrm{rad}$ for the vertical and $\approx 0.08$ rad for the horizontal direction for an interrogated sensor length of $0.8 \mathrm{~m}$ and over an interferometric bandwidth of $21 \mathrm{kHz}$. Furthermore, it was confirmed by Fourier analysis that the noise floor is generally flat, resulting in worst case interrogation noise levels for the vertical direction of $1.2 \mathrm{mrad} \cdot \mathrm{Hz}^{-0.5}$. The reason for the difference between the measurements for the two directions is likely to be the return signal strength of the participating in-fiber reflectors used for each measurement, as the signal strengths exhibited in Fig. 3 are not yet fully in the phase-noise limited region [26] for all reflectors. Using the nominal value of the slope angle sensitivity of $487 \frac{\mathrm{rad}}{\circ}$, this equates to a noise standard deviation of $0.35 \cdot 10^{-3} \circ$ in the slope angle signal, corresponding to a noise level of $2.4 \cdot 10^{-6} \circ \cdot \mathrm{Hz}^{-0.5}$ for the worst case vertical slope angle measurement. Displacement noise for the worst case vertical movements can then be obtained from the lower panel in Fig. 7(b), where the displacement noise standard deviation of $0.56 \mu \mathrm{m}$ can be extracted for both linear and spline interpolation cases. Over the $200 \mathrm{~Hz}$ bandwidth used, this equates to displacement noise levels of $40 \mathrm{~nm} \cdot \mathrm{Hz}^{-0.5}$ at the cantilever tip.

\section{Further Discussion}

In the FOSS approach presented in this paper, as previously discussed, the use of a custom extruded PTFE profile with a directional guide as a sensor rod would improve the robustness of this approach by removing uncertainties of the sensor rod orientation, potentially eliminating the need for the directional calibration performed in Section IV-A. This would also reduce considerably the uncertainty in the value of the lateral core displacement $\delta$ and ultimately of the slope angle sensitivity $\Delta \phi \cdot \psi^{-1}$. For the nominal value of $\delta=3.0 \mathrm{~mm}$ and the sensor rod length of $l=0.8 \mathrm{~m}$ used, the curvature sensitivity $\Delta \phi \cdot \kappa^{-1}$, a widely used quantity when comparing FOSS approaches, is $22.3 \cdot 10^{3} \mathrm{rad} \cdot \mathrm{m}$ using (3). The use of multicore fiber would also be possible using this approach, resulting in considerably smaller sensor rod protrusion diameters, but also reducing the curvature sensitivity by a factor of 60 for a typical multicore fiber of $\delta=50 \mu \mathrm{m}$ lateral core spacing. In the static deflection experiment shown in Fig. 6(d) it became clear that an increase in spatial resolution of the FOSS measurement would be beneficial in cases of rapidly changing curvature profiles. Based on previous experience of the wavelength tuning capabilities of diode lasers, an improvement in the spatial resolution by up to a factor of four is deemed possible using DFB-type diode lasers. However, keeping the overall sensor rod length constant at an increased resolution while maintaining the same data acquisition bandwidth of the interrogation system would also entail a corresponding reduction in the interferometric bandwidth that can be recorded [26]. A further point not yet addressed is the polarisation sensitivity inherent in any long-gauge length interferometric technique, potentially leading to polarisation-induced signal fading. It is expected that the system should show a considerable robustness against any polarisation-induced signal fading after fabrication as all desired interferences occur within each fiber string, however, if this poses a problem polarisation-diversity detection [36] could also be employed.

\section{Conclusion}

The theory of spatially-resolved interferometric fiber-optic shape sensing has been presented, showing that the parameter measured using this approach is slope angle sampled at the respective sensor section end positions. Fiber-optic shape sensing using fiber segment interferometry was demonstrated on a metal cantilever. A flexible PTFE sensor rod with lateral fiber core separation of $3.0 \mathrm{~mm}$ was fabricated and 20 in-fiber Bragg reflectors were inscribed and multiplexed using a single fullyenclosed interrogation unit. Slope angle measurements at four sensor sections at a spatial resolution of $20 \mathrm{~cm}$ could be recorded over an interferometric bandwidth of $21 \mathrm{kHz}$. Using free-space interferometric verification measurements it was shown that the errors in the calculation of the cantilever tip displacement from the measured slope angle data do not exceed $10 \%$ for typical vibration patterns using either linear or a cubic spline interpolation approaches that do not make any assumptions on the underlying structure. For a sensor of nominal curvature sensitivity of $22.3 \cdot 10^{3} \mathrm{rad} \cdot \mathrm{m}$ and worst-case interrogation phase noise levels of $1.2 \mathrm{mrad} \cdot \mathrm{Hz}^{-0.5}$, slope angle measurements at noise levels of $2.4 \cdot 10^{-6} \cdot \mathrm{Hz}^{-0.5}$ could be recorded, equating, for the specific cantilever test object of length $0.8 \mathrm{~m}$, to tip displacement noise levels of $40 \mathrm{~nm} \cdot \mathrm{Hz}^{-0.5}$. This performance clearly demonstrates the potential of this sensing concept for highly sensitive and cost-effective fiber-optic shape, lateral displacement or vibration measurements for structural health monitoring and further applications.

\section{ACKNOWLEDGMENT}

The underlying data can be accessed from the Cranfield Online Research Data Repository at http://dx.doi.org/10.17862/ cranfield.rd.5209120. 


\section{REFERENCES}

[1] H. J. Patrick, C. Chang, and S. T. Vohra, "Long period fibre gratings for structural bend sensing," Electron. Lett., vol. 34, no. 18, pp. 1773-1775, 1998.

[2] C. C. Ye, S. W. James, and R. P. Tatam, "Simultaneous temperature and bend sensing with long-period fiber gratings," Opt. Lett., vol. 25, no. 14, pp. 1007-1009, 2000.

[3] P. M. Blanchard et al., "Two-dimensional bend sensing with a single, multi-core optical fiber," Smart Mater. Struct., vol. 9, no. 2, pp. 132-140, 2000

[4] M. J. Gander et al., "Two-axis bend measurement using multicore optical fiber," Opt. Commun., vol. 182, no. 1, pp. 115-121, 2000.

[5] M. J. Gander et al., "Bend measurement using Bragg gratings in multicore fibre," Electron. Lett., vol. 36, no. 2, pp. 120-121, 2000

[6] G. M. H. Flockhart, W. N. Macpherson, J. S. Barton, and J. D. C. Jones, "Two-axis bend measurement with Bragg gratings in multicore optical fiber," Opt. Lett., vol. 28, no. 6, pp. 387-389, 2003.

[7] T. Allsop et al., "Bending and orientational characteristics of long period gratings written in D-shaped optical fiber [directional bend sensors]," IEEE Trans. Instrum. Meas., vol. 53, no. 1, pp. 130-135, Feb. 2004.

[8] W. N. MacPherson et al., "Tunnel monitoring using multicore fibre displacement sensor," Meas. Sci. Technol., vol. 17, no. 5, pp. 1180-1185, 2006.

[9] R. G. Duncan et al., "High-accuracy fiber-optic shape sensing," Proc. SPIE, Sensor Syst. Netw., vol. 6530, pp. 65301S-1-65301S-11, 2007.

[10] S. Rapp, L. H. Kang, J. H. Han, U. C. Mueller, and H. Baier, "Displacement field estimation for a two-dimensional structure using fiber Bragg grating sensors," Smart Mater. Struct., vol. 18, no. 2, pp. 25006-1-25006-12, 2009

[11] O. Frazão et al., "All Fiber Mach-Zehnder interferometer based on suspended twin-core fiber," IEEE Photon. Technol. Lett., vol. 22, no. 17, pp. 1300-1302, Sep. 2010

[12] M. Deng, C. P. Tang, T. Zhu, and Y. J. Rao, "Highly sensitive bend sensor based on Mach-Zehnder interferometer using photonic crystal fiber," Opt. Commun., vol. 284, no. 12, pp. 2849-2853, 2011.

[13] H. Bang, H. Kim, and K. Lee, "Measurement of strain and bending deflection of a wind turbine tower using arrayed FBG sensors," Int. J. Precis. Eng. Manuf., vol. 13, no. 12, pp. 2121-2126, 2012.

[14] J. P. Moore and M. D. Rogge, "Shape sensing using multi-core fiber optic cable and parametric curve solutions," Opt. Express, vol. 20, no. 3, pp. 2967-2973, 2012

[15] R. Wang et al., "Highly sensitive curvature sensor using an in-fiber MachZehnder interferometer," IEEE Sensors J., vol. 13, no. 5, pp. 1766-1770, May 2013.

[16] R. J. Roesthuis, M. Kemp, J. J. van den Dobbelsteen, and S. Misra, "Three-dimensional needle shape reconstruction using an array of fiber Bragg grating sensors," IEEE/ASME Trans. Mechatronics, vol. 19, no. 4, pp. 1115-1126, Aug. 2013

[17] R. Xu, A. Yurkewich, and R. V. Patel, "Curvature, torsion, and force sensing in continuum robots using helically wrapped FBG sensors," IEEE Robot. Autom. Lett., vol. 1, no. 2, pp. 1052-1059, Jul. 2016.

[18] M. J. Nicolas, R. W. Sullivan, and W. L. Richards, "Large scale applications using FBG sensors: Determination of in-flight loads and shape of a composite aircraft wing," Aerospace, vol. 3, no. 3, pp. 18-1-18-15, 2016

[19] Z. Zhao, M. A. Soto, M. Tang, and L. Thévenaz, "Curvature and shape distributed sensing using Brillouin scattering in multi-core fibers," in Proc. Adv. Photon. Congr., Vancouver, Canada, 2016, pp. SeM4D.4-1SeM4D.4-3.
[20] T. Kissinger, E. Chehura, S. W. James, and R. P. Tatam, "Multiplexing curvature sensors using fibre segment interferometry for lateral vibration measurements," in Proc. 25th Opt. Fiber Sensors Conf., Jeju, South Korea, 2017, pp. 1-4.

[21] K. O. Hill and G. Meltz, "Fiber Bragg grating technology fundamentals and overview," J. Lightw. Technol., vol. 15, no. 8, pp. 1263-1276, Aug. 1997.

[22] B. Lee, "Review of the present status of optical fiber sensors," Opt. Fiber Technol., vol. 9, no. 2, pp. 57-79, 2003.

[23] B. A. Childers et al., "Use of 3000 Bragg grating strain sensors distributed on four eight-meter optical fibers during static load tests of a composite structure," in Proc. Smart Structures Mater., Newport Beach, CA, USA, vol. 4332, pp. 133-142, 2001.

[24] K. Yüksel, V. Moeyaert, P. Mégret, and M.Wuilpart, "Complete analysis of multireflection and spectral-shadowing crosstalks in a quasi-distributed fiber sensor interrogated by OFDR," IEEE Sensors J., vol. 12, no. 5 , pp. 988-995, May 2012.

[25] S. J. Rothberg et al., "An international review of laser Doppler vibrometry: Making light work of vibration measurement," Opt. Laser Eng., to be published. doi: 10.1016/j.optlaseng.2016.10.023.

[26] T. Kissinger, R. Correia, T. O. H. Charrett, S. W. James, and R. P. Tatam, "Fiber segment interferometry for dynamic strain measurements," J. Lightw. Technol., vol. 34, no. 19, pp. 4620-4626, Oct. 2016.

[27] T. Kissinger, T. O. H. Charrett, and R. P. Tatam, "Range-resolved interferometric signal processing using sinusoidal optical frequency modulation," Opt. Express, vol. 23, no. 7, pp. 9415-9431, 2015.

[28] C. Polhemus, "Two-wavelength interferometry," Appl. Opt., vol. 12, no. 9 pp. 2071-2074, 1973.

[29] C. D. Butter and G. B. Hocker, "Fiber optics strain gauge," Appl. Opt., vol. 17 , no. 18 , pp. 2867-2869, 1978.

[30] T. Kissinger, T. O. H. Charrett, and R. P. Tatam, "Fibre segment interferometry using code-division multiplexed optical signal processing for strain sensing applications," Meas. Sci. Technol., vol. 24, no. 9, pp. 940111-94011-13, 2013.

[31] J. M. Gere and B. Goodno, Mechanics of Materials, 9th ed. Boston, MA, USA: Cengage Learning, 2016.

[32] M. Jacobsen, D. Richmond, M. Hogains, and R. Kastner, "RIFFA 2.1: A reusable integration framework for FPGA accelerators," ACM Trans. Reconfigurable Technol., vol. 8, no. 4, pp. 22-1-22-23, 2015.

[33] Z. Wang, F. Shen, L. Song, X. Wang, and A. Wang, "Multiplexed fiber Fabry-Perot interferometer sensors based on ultrashort Bragg gratings," IEEE Photon. Technol. Lett., vol. 19, no. 8, pp. 622-624, Apr. 2007.

[34] J. Roths and F. Jülich, "Determination of strain sensitivity of free fiber Bragg gratings," in Proc. Opt. Sensors Conf., Strasbourg, France, vol. 7003, pp. 700308-1-700308-8, 2008.

[35] T. Kissinger, T. O. H. Charrett, S. W. James, A. Adams, A. Twin, and R. P. Tatam, "Simultaneous laser vibrometry on multiple surfaces with a single beam system using range-resolved interferometry," SPIE Opt. Metrol., Munich, Germany, vol. 9525, pp. 952520-1-952520-7, 2015.

[36] N. J. Frigo, A. D. Dandridge, and A. B. Tveten, "Technique for elimination of polarisation fading in fibre interferometers," Electron. Lett., vol. 20 , no. 8, pp. 319-320, 1984.

Authors' biographies not available at the time of publication. 
2017-09-12

\section{Dynamic fiber-optic shape sensing using fiber segment interferometry}

Kissinger, Thomas

IEEE

Kissinger T, Chehura E, Staines SE, et al., (2018) Dynamic fiber-optic shape sensing using fiber segment interferometry. Journal of Lightwave Technology, Volume 36, Issue 4, February 2018, pp. 917-925

http://dx.doi.org/10.1109/JLT.2017.2750759

Downloaded from Cranfield Library Services E-Repository 